\title{
Band dispersion and electronic lifetimes in crystalline organic semiconductors
}

\author{
S. Ciuchi ${ }^{1}$ and S. Fratini ${ }^{2}$ \\ ${ }^{1}$ Istituto dei Sistemi Complessi CNR, CNISM and Dipartimento di Fisica, \\ Università dell'Aquila, via Vetoio, I-67100 Coppito-L'Aquila, Italy \\ 2 Institut Néel-CNRS and Université Joseph Fourier, \\ Bô̂te Postale 166, F-38042 Grenoble Cedex 9, France
}

(Dated: September 24, 2018)

\begin{abstract}
The consequences of several microscopic interactions on the photoemission spectra of crystalline organic semiconductors (OSC) are studied theoretically. It is argued that their relative roles can be disentangled by analyzing both their temperature and their momentum/energy dependence. Our analysis shows that the polaronic thermal band narrowing, that is the foundation of most theories of electrical transport in OSC, is inconsistent in the range of microscopic parameters appropriate for these materials. An alternative scenario is proposed to explain the experimental trends.

PACS numbers:
\end{abstract}

Introduction. It is now becoming possible to measure Angle-Resolved photoemission (ARPES) spectra of organic semiconductors (OSC) with increasingly high accuracy, revealing intrinsic properties of conduction electrons in these materials. Clear energy-momentum dispersions, indicative of the formation of well-defined electronic bands, have been reported in pentacene monolayers [1], crystalline films [2, 3] as well as rubrene single crystals [4]. Since photoemission probes the very nature of electronic excitations, the measured spectra (in particular, their deviations from non-interacting Bloch bands) provide direct information on the interactions affecting the electron motion. Ultimately, such information could help establishing a proper microscopic model for the electron dynamics in these materials. For this procedure to be viable, however, one must be able to provide reliable predictions from the theoretical side, identifying characteristic signatures of the different microscopic phenomena at work. Only then the comparison with the experimental ARPES spectra can be effectively used to sort out the relevant microscopic interactions in crystalline OSC.

In this work, we consider the effect of several microscopic interactions that have been considered to play a role in crystalline OSC: (i) the coupling of electrons to intra-molecular vibrations; (ii) dynamical disorder originating from thermal inter-molecular motions; (iii) static disorder, of both chemical and structural origin, giving rise to spatial variations of the molecular site energies and (iv) details of the electronic structure, most importantly the features arising from the non-equivalence of the molecules in the unit cell. On general grounds, these interactions can affect either the band dispersion, the electronic lifetimes (i.e. respectively the position and width of the ARPES peaks), or both. In order to disentangle all these effects experimentally, it is important to characterize their temperature dependences: for example, (i, ii) can in principle depend on temperature, via the thermal changes in the phonon population while (iii,iv) should be essentially temperature independent, being de- termined by the structural characteristics of the sample. A similar assessment can be made concerning their momentum/energy dependence, i.e. how different interactions affect distinct regions of the electronic spectrum. Our aim here is to clarify and quantify these aspects by performing a reliable calculation of the electronic spectral function in a controlled microscopic model.

Model. We consider the following Hamiltonian,

$$
\begin{aligned}
H=H^{(0)} & +H^{(i)}+H^{(i i)}+H^{(i i i)}+H^{(i v)}+H^{(v i b)} \\
H^{(0)} & =-J \sum_{j}\left(c_{j}^{+} c_{j+1}+c_{j+1}^{+} c_{j}\right) \\
H^{(i)} & =\sum_{j} g c_{j}^{+} c_{j} X_{j} \\
H^{(i i)} & =-\sum_{j} f\left(u_{j}-u_{j+1}\right)\left(c_{j}^{+} c_{j+1}+c_{j+1}^{+} c_{j}\right) \\
H^{(i i i)} & =\sum_{j} \epsilon_{j} c_{j}^{+} c_{j} ; H^{(i v)}=\delta \sum_{j}(-1)^{j} c_{j}^{+} c_{j} \\
H^{(v i b)} & =\sum_{j} \frac{M \Omega_{0}^{2} X_{j}^{2}}{2}+\frac{P_{j}^{2}}{2 M}+\frac{m \omega_{0}^{2} u_{j}^{2}}{2}+\frac{p_{j}^{2}}{2 m} .
\end{aligned}
$$

$H^{(0)}$ describes the tight binding motion of holes in a HOMO band that we take to be one dimensional for simplicity, reflecting the marked anisotropy that is commonly observed in crystalline OSC. $H^{(i)}$ and $H^{(i i)}$ are respectively the "Holstein" interaction with intramolecular deformations $X_{j}$ of frequency $\Omega_{0}$, with coupling strength $g$, and the "Peierls" or "Su-SchriefferHeeger" (SSH) interaction with molecular displacements $u_{j}$ of frequency $\omega_{0}$. These two interactions modulate respectively the molecular energies and the inter-molecular transfer integrals. For the latter we assume a linear dependence on the inter-molecular distance, $f\left(u_{j}-u_{j+1}\right)=$ $J \alpha_{S S H}\left(u_{j}-u_{j+1}\right)$, being $\alpha_{S S H}$ the coupling strength. $H^{(i i i)}$ describes disordered site energies $\epsilon_{j}$ obeying a gaussian distribution of fixed width $\Delta$, and $H^{(i v)}$ an alternating potential $\pm \delta$ reflecting a non-equivalence of 
the sites in the unit cell, as is commonly observed in OSC. $H^{(v i b)}$ is the Hamiltonian for free harmonic vibrations. In the following we set $\hbar=k_{B}=1$ and express the strength of the electron-vibration interactions by introducing the dimensionless parameters $\alpha_{H}=g / \Omega_{0}$ and $\lambda_{S S H}=\alpha_{S S H}^{2} J /\left(2 M \omega_{0}^{2}\right)$. Typical bandwidts in organic semiconductors are $W \sim 0.5 \mathrm{eV}$, corresponding to $J \sim 0.125 \mathrm{eV}$ in our one-dimensional model. The microscopic parameters that can be found in the literature are $\omega_{0} \simeq(0.05-0.1) J$ and $\Omega_{0} \simeq(1-2) J$ with $\lambda_{S S H} \simeq$ $0.15-0.3$ and $\alpha_{H}^{2} \simeq 0.2-0.5$, and $\delta=(0.1-0.3) \mathrm{J}$, $\Delta=(0.1-0.3) J[1,4,10-16]$.

Method. Owing to the low frequency of the intermolecular vibrations, that stems from the large molecular mass, we have both $\omega_{0} \ll J$ and $\omega_{0} \lesssim T$ in the relevant temperature range. In this regime the displacements $u_{j}$ in $H^{(i i)}$ can be treated as a random field obeying a gaussian distribution, causing a statistical disorder in the inter-molecular transfer integrals [5]. The solution of all the terms in Eq. (11) but $H^{(i)}$ therefore reduces to a problem of non-interacting electrons in the presence of both "site-diagonal" and "off-diagonal" disorder, that can be efficiently treated with the method described in Ref. [6]. Instead, the full quantum nature of the intramolecular vibrations must be retained in $H^{(i)}$, because $T \ll \Omega_{0}$. To this aim we devise a method of solution that provides reliable results in the case $\Omega_{0} \gtrsim J$, which includes the moderate vibrational frequency regime of interest here, $\Omega_{0} \simeq(1-2) J$. As we show below, this treatment of the intra-molecular electron-vibration interactions can be straightforwardly implemented in the disordered environment provided by the remaining terms in the Hamiltonian.

The Green's function of the problem can be defined in the site representation as

$$
G_{i, j}=\int \Pi_{l} d u_{l} \Pi_{m} d \epsilon_{m} P(\mathbf{u}) P_{d i s}(\boldsymbol{\epsilon}) G_{i, j}(\mathbf{u}, \boldsymbol{\epsilon})
$$

where $P(\mathbf{u}) \propto \Pi_{l} \exp \left(-u_{l}^{2} / 2 \sigma^{2}\right)$ is the statistical distribution of local displacements $\mathbf{u}=\left\{u_{l}\right\}$, with $\sigma^{2}=\left[2 M \omega_{0} \tanh \left(\omega_{0} / 2 T\right)\right]^{-1}$ and $P_{d i s}(\boldsymbol{\epsilon}) \propto$ $\Pi_{m} \exp \left(-\epsilon_{m}^{2} / 2 \Delta^{2}\right)$ is the distribution of local energies $\boldsymbol{\epsilon}=\left\{\epsilon_{m}\right\}$. For a given set of inter-molecular deformations and disorder variables the Green's function of the electronic problem $H_{e l}=H-H^{(i)}$ in the absence of the Holstein term can be obtained as an inversion of a tridiagonal matrix in the site representation: $G_{i, j}(\mathbf{u}, \boldsymbol{\epsilon})=$ $\left[\left(\omega-H_{e l}\right)^{-1}\right]_{i, j}$ with [6]

$$
\omega-H_{e l}=\left(\begin{array}{cccc}
a_{0}^{(0)} & b_{0} & 0 & \cdots \\
b_{0} & a_{1}^{(0)} & b_{1} & \cdots \\
\cdots & \cdots & \cdots & \cdots \\
\cdots & 0 & b_{N-1} & a_{N}^{(0)}
\end{array}\right)
$$

The interaction term $H^{(i)}$ is subsequently included at a local level. In practice, we consider a thermalized Holstein impurity at each site $i$, whose Green's function is defined as

$$
G_{l o c}^{(i)}(\omega)=\frac{1}{Z_{H}}\left[\sum_{n=0}^{\infty} \frac{e^{-n \Omega_{0} / T}}{\mathcal{G}_{i, i}^{-1}(\omega)-\Sigma_{H, i}^{e m,(n)}-\Sigma_{H, i}^{e m,(n)}}\right]
$$

with $Z_{H}=\sum_{n=0}^{\infty} e^{-n \Omega_{0} / T}$ [we drop the indices $(\mathbf{u}, \boldsymbol{\epsilon})$ in the following]. Here $\mathcal{G}_{i, i}$ is the local propagator obtained from the inversion of the matrix Eq. (8) and $\Sigma_{H, i}^{e m,(n)}$, $\Sigma_{H, i}^{e m,(n)}$ are the local emission/absorption self-energies in the n-phonon propagator of the impurity. These can be expressed as continued fractions [7]:

$$
\begin{aligned}
\Sigma_{H, i}^{e m,(n)}(\omega) & =(n+1) g^{2} / \mathcal{G}_{i, i}^{-1}\left[\omega-\Omega_{0}\right]-(n+2) g^{2} / \mathcal{G}_{i, i}^{-1}\left[\omega-2 \Omega_{0}\right]-\ldots \quad ; n \geq 0 \\
\Sigma_{H, i}^{a b s,(n)}(\omega) & =n g^{2} / \mathcal{G}_{i, i}^{-1}\left[\omega+\Omega_{0}\right]-(n-1) g^{2} / \mathcal{G}_{i, i}^{-1}\left[\omega+2 \Omega_{0}\right]-\ldots \quad ; n \geq 1,
\end{aligned}
$$

the latter fraction obviously ending at $n$-th stage. To evaluate the Green's function $G_{i, j}(\mathbf{u}, \boldsymbol{\epsilon})$ of the full lattice problem, we now invert Eq. (8) with the replacement $a_{i}^{(0)}=\omega-\left[H_{e l}\right]_{i, i} \longrightarrow a_{i}=\omega-\left[H_{e l}\right]_{i, i}-\Sigma_{H, i}$ where

$$
\Sigma_{H, i}=\left(\mathcal{G}_{i, i}\right)^{-1}-\left(G_{l o c}^{(i)}\right)^{-1}
$$

is the local self-energy of the Holstein-impurity problem at each site $i$ [the off-diagonal terms $b_{i}=J+f\left(u_{i}-u_{i+1}\right)$ are kept unchanged]. This treatment of the Holstein electron-vibration coupling has formally the same structure as Dynamical Mean Field Theory [7], except for the absence of a self-consistency loop. It reduces to the $\mathrm{MA}^{(0)}$ approximation of Refs. 8, 9] at $T=0$ and, as in $\mathrm{MA}^{(0)}$, it is most appropriate in the non-adiabatic regime, $\Omega_{0} \gtrsim J$.

The above Eqs. (9)-(12) solve the Holstein interaction problem for any given configuration of the diagonal and off-diagonal disorder. The solution of the full Hamilto- 
a)

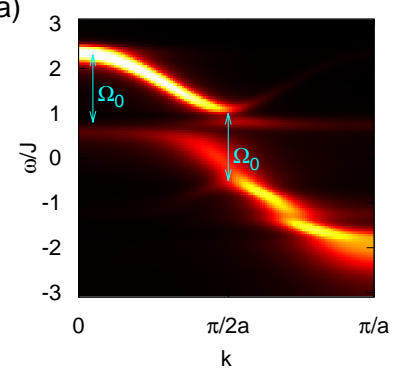

b)

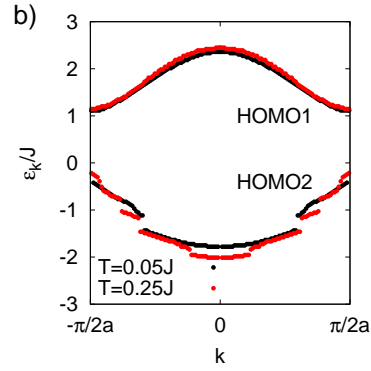

FIG. 1: (a) Spectral function $A(k, \omega)$ for a hole in a HOMO band, calculated for $J=0.125 \mathrm{eV}, \lambda_{S S H}=0.2, \omega_{0}=0.05 \mathrm{~J}$, $\alpha_{H}^{2}=0.33, \Omega_{0}=1.5 \mathrm{~J}, \delta=0.3 \mathrm{~J}, \Delta=0.2 \mathrm{~J}$ at $T=0.05 \mathrm{~J}$. (b) Position of the maxima, tracking the renormalized band dispersion $\varepsilon_{k}$ at low and room temperature (reduced Brillouin zone).

nian Eq. (1) is obtained from Eq. (7) upon averaging over 50000 realizations of disorder variables on a chain of $N=512$ sites. The spectral function is $A(k, \omega)=$ $-\frac{1}{\pi} \operatorname{Im} G(k, \omega)$, where $G(k, \omega)=\frac{1}{N^{2}} \sum_{i, j} e^{i k a(i-j)} G_{i, j}(\omega)$ is the Green's function in momentum space.

Results. Fig. 17 shows a photoemission spectrum calculated with the above procedure for holes in a onedimensional HOMO band, ideally representing the direction of maximum conduction in an organic crystal, for a representative choice of parameters [slight changes in the parameter values within the range indicated after Eqs. (1)-(6) do not appreciably modify the scenario]. To understand the results it is instructive to analyze the different terms in the Hamiltonian separately. We start from the interaction with the intra-molecular vibrations, $H^{(i)}$, that is responsible for the most prominent features observed in the spectrum. As is well known, in the case of isolated molecules this term gives rise to "shakeoff" satellites of the molecular levels, appearing at multiples of the vibrational frequency $\Omega_{0}$ [10]. The number of visible satellites is set by the coupling strength, $N_{v i b}=\alpha_{H}^{2}$, and is indicative of the amount of vibrational quanta constituting the polaronic deformation.

In a crystalline environment the situation is more complex. The molecular picture is only recovered when the electronic band dispersion is small compared to the vibration frequency, $W \ll \Omega_{0}$. This however does not apply to organic crystals, where despite the arguably narrow bandwidths as compared to inorganic semiconductors, $W$ is still the largest energy scale in the problem. Being $W>\Omega_{0}$, the periodic overtones characteristic of molecular spectra are replaced by cuts in the band dispersion. These features are analogous to the "kinks" that are commonly observed in the photoemission spectra of solids with sizable electron-boson coupling. They add a vibrational fine structure to the spectrum [17] without
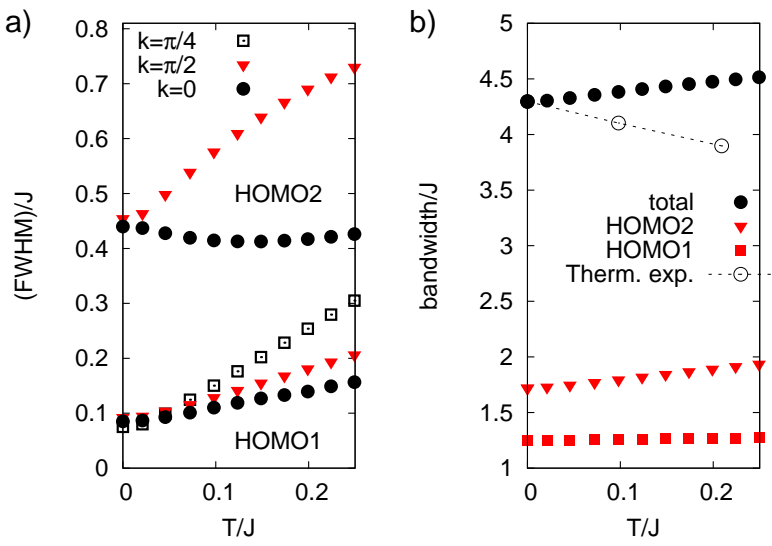

FIG. 2: (a) Temperature dependence of the spectral linewidths at different points in the BZ, for the same parameters as in Fig. 1. (b) Widths of the individual subbands and total bandwidth. Open circles are obtained including the lattice thermal expansion calculated in Ref. 24] (the bandwidth is normalized to the zero-temperature value of $J$ ).

much affecting the overall bandwidth [7, 8, 18]. This phenomenon is clearly visible in Fig. 1a, where the cosine dispersion starting from the top of the band is cut out by phonon resonances at $\Omega_{0}$ and $2 \Omega_{0}$ (indicated by arrows). In the case $\Omega_{0} \sim(1-2) J$ that is relevant to OSC, the interaction with intra-molecular vibrations effectively splits the HOMO dispersion into two main subbands separated by a sizable direct gap, as shown in Fig. 1b. The gap opens up where the dispersion crosses the first vibrational cut, which falls accidentally around $k \sim \pi / 2$. This result can explain the large separation between the two HOMO subbands of pentacene 2, 3], that is one order of magnitude larger than that predicted by ab-initio calculations on accounts of the structural non-equivalence in the unit cell [15, 19].

Fig. 2a reports the lifetimes for states at different points of the Brillouin zone (BZ). The large difference between the HOMO1 and HOMO2 branches, that was also observed in Ref. 2], is an additional distinctive feature of the intra-molecular interaction $H^{(i)}$ : according to the arguments given above, the HOMO1 band lies by construction below the threshold for the emission of a vibrational quantum, $\left|\varepsilon_{k}-\varepsilon_{k=0}\right|<\Omega_{0}$, and therefore the electronic lifetime there is mostly insensitive to the effects of intramolecular vibrations [7, 8]. Vibronic scattering processes are instead allowed in the second subband, where they cause a much larger line broadening, with linewidths of the order of the electronic transfer rate itself. Since only the HOMO1 states near the band edge can be thermally populated or doped in a field-effect device, we argue that scattering from high-frequency vibrations should not play a predominant role in the transport mechanism of OSC.

The effects related to $H^{(i)}$ are essentially temperature independent, because the considered vibrations cannot 
be thermally excited in the relevant range $T \ll \Omega_{0}$. The temperature dependence observed in Fig. 2a therefore arises from other microscopic mechanisms present in the model. While the static disorder, $H^{(i i i)}$, can be excluded (it adds a constant broadening $\propto \Delta$ to the spectral lines), the scattering from slow inter-molecular lattice vibrations in $H^{(i i)}$ is indeed strongly temperature-dependent: because the lattice motions are thermally excited for $T \gtrsim \omega_{0} / 2$, the linewidths increase linearly with temperature, which is indeed observed in the HOMO1 sector of Fig. 2a. Different slopes arise at different points of the BZ because the scattering from inter-molecular lattice vibrations is minimum at the band edges [6].

Finally, Fig. 2b illustrates the temperature dependence of the calculated electronic bandwidths. The total HOMO width (black dots) exhibits a moderate increase with temperature, that is entirely caused by the coupling to low-frequency inter-molecular vibrations: the thermal fluctuations of the lattice structure lead to an average increase of the transfer integrals given by $J_{a \nu}^{2}=J^{2}+\sigma_{J}^{2}$, with $\sigma_{J}^{2}=4 \lambda_{S S H} J T$ for $T \gtrsim \omega_{0} / 2[6,14,[20,21]$. The coupling to low-frequency intra-molecular vibrations, not considered here, would also lead to an analogous effect 22]. Importantly, this trend is inverted if one takes into account the large thermal expansion coefficient characteristic of organic crystals (open circles in Fig. 2b) 23] : upon heating, the lattice expansion leads to an effective reduction of the "bare" transfer integral $J$ entering in Eq. (2) 24]. The resulting bandwidth reduction quantitatively agrees with the thermal band narrowing reported in pentacene [2, 25]. This effect should not be confused with the the phenomenon of polaronic band narrowing [26], that is the foundation of popular theories of electrical transport in crystalline OSC, but is inconsistent with the parameter range relevant to these materials.

In standard polaron theories [11, 26, 27], that build on the molecular limit $W \ll \Omega_{0}$, the dressing of charge carriers by fast molecular vibrations causes a renormalization of the transfer integrals and a consequent reduction of the bandwidths, which is exponential in the phonon number $N_{v i b}$. Upon increasing the temperature the "polaronic" band further shrinks due to the increased population of molecular vibrations, following $W \rightarrow W e^{-N_{v i b}(T)}$, with $N_{v i b}(T)=\alpha_{H}^{2}\left[1+2 n_{B}(T)\right]$ and $n_{B}(T)$ the Bose factor. For a measurable effect to be observed at room temperature, however, one would require $\Omega_{0} \lesssim 1000 \mathrm{~K} \simeq 90 \mathrm{meV}$. Such value is considerably smaller than the bandwidth and therefore contradicts the molecular limit assumption.

In the regime of moderate coupling strengths and $\Omega_{0}<W$ of interest for OSC, it is the very hypothesis of an exponential renormalization that fails [8, 18, 22]. A polaronic band can still be identified - it is nothing but the HOMO1 band of Fig.1 - but its width is now of the order of the vibration frequency $\Omega_{0}$, and not governed by the textbook exponential relation. If we now focus on the entire HOMO dispersion, the absence of polaronic band narrowing is even more evident: with the present parameters one would predict a $30 \%$ polaronic reduction of the bandwidth from the non-interacting value $W=4 J$, which is clearly not observed in Fig. 1 (we show in the suplementary EPAPS file that this is not a drawback of the present method). The physical reason for the failure of the conventional picture is that the molecular degrees of freedom are not fast enough to rearrange during the electron motion, and therefore cannot provide the instantaneous renormalization implied in the molecular limit.

Concluding remarks. The calculations presented here show that the photoemission spectra of crystalline organic semiconductors can differ significantly from the non-interacting band picture, reflecting a sizable coupling of the conduction electrons with other degrees of freedom in the system. The most salient features in the spectra come from the interaction with high-frequency intra-molecular vibrations, that causes the opening of apparent gaps in the electronic dispersion at multiples of the vibrational energy $\Omega_{0}$. However, such high-frequency modes are unable to efficiently scatter the quasiparticle states at the lowest binding energies. For this reason, the origin of the temperature dependence of the mobility in these materials cannot be explained by polaronic effects but should rather be seeked in the interaction with inter-molecular vibrational modes of much lower frequency, whose temperature-dependent scattering rates are directly accessible in ARPES experiments.

We thank A. Girlando for enlightening discussions.

[1] H. Kakuta et al., Phys. Rev. Lett. 98, 247601 (2007)

[2] R. C. Hatch, D. L. Huber and H. Hochst, Phys. Rev. Lett. 104, 047601 (2010)

[3] R. C. Hatch, D. L. Huber and H. Hochst, Phys. Rev. B 80, 081411(R) (2009)

[4] S. Machida et al., Phys. Rev. Lett. 104, 156401 (2010)

[5] While the disorder dynamics seem to be crucial to the transport mechanism 21] [see also S. Ciuchi et al, arXiv:1010.2893, they can be safely neglected in the evaluation of the spectral function.

[6] S. Fratini \& S. Ciuchi, Phys. Rev. Lett. 103, 266601 (2009)

[7] S. Ciuchi, F. de Pasquale, S. Fratini, and D. Feinberg, Phys. Rev. B 56, 4494 (1997)

[8] G. L. Goodvin, M. Berciu \& G. Sawatzky, Phys. Rev. B 74,245104 (2006)

[9] M. Berciu, A. S. Mishchenko and N. Nagaosa, EPL, 89, 37007 (2010)

[10] V. Coropceanu et al., Phys. Rev. Lett. 89, 275503 (2002)

[11] K. Hannewald \& P. A. Bobbert. Phys. Rev. B 69, 075212 (2004).

[12] D. A. da Silva Filho, E.-G. Kim, and J.-L. Brédas, Adv. Mater. 17, 1072 (2005)

[13] A. Girlando, et al., Phys. Rev. B 82, 035208 (2010)

[14] R. S. Sanchez-Carrera et al, J. Am. Chem. Soc. 132, 14437 (2010) 
[15] H. Yoshida, N. Sato, Phys. Rev. B 77, 235205 (2008)

[16] W. L. Kalb, et al. Phys. Rev. B 81, 155315 (2010)

[17] H. Yamane et al., Phys. Rev. B 72, 153412 (2005)

[18] M. Hohenadler, M. Aichhorn, and W. von der Linden, Phys. Rev. B 68, 184304 (2003)

[19] The large HOMO1/HOMO2 gap in Fig. 1 remains unchanged if we set $\delta=0$. The effect of the structural term $H^{(i v)}$ reduces to the small splitting visible at $k=\pi / 2$ around $\omega \simeq J$ and minor features in the HOMO2 band.

[20] P. Gosar \& S.-I. Choi, Phys. Rev. 150, 529 (1966)
[21] A. Troisi \& D. L. Cheung, J. Chem. Phys. 131, 014703 (2009)

[22] S. Fratini \& S. Ciuchi, Phys. Rev. B 72, 235107 (2005)

[23] S. Haas et al., Phys. Rev. B 76, 205203 (2007)

[24] M. Masino, et al., Macromol. Symp. 212, 375 (2004)

[25] N. Koch et al., Phys. Rev. Lett. 96, 156803 (2006)

[26] T. Holstein, Ann. Phys. 8, 343 (1959)

[27] V. Coropceanu, et al, Chem. Rev. 107, 926 (2007) 